\title{
Thoracic meningocele in lumbo-costo-vertebral syndrome in a child: possible enlargement with repeated motion by anchoring to the diaphragm
}

\author{
Takafumi Wataya, MD, PhD, Kyohei Horikawa, MD, Masashi Kitagawa, MD, and \\ Yuzuru Tashiro, MD, PhD \\ Department of Neurosurgery, Shizuoka Children's Hospital, Shizuoka, Japan
}

Lumbo-costo-vertebral syndrome (LCVS) is a rare disorder in children that is characterized by hemivertebrae, congenital absence of ribs, meningocele, and hypoplasia of the truncal and abdominal wall presenting as a congenital lumbar hernia. An otherwise healthy 12-month-old girl was referred to the authors' hospital with soft swelling on her left middle back; scoliosis had been present since birth. Imaging revealed a thoracic meningocele, ectopia of the spleen suggesting lumbar hernia, multiple anomalies of the thoracic vertebral columns, and defects of the ribs; thus, LCVS was diagnosed. Surgical observation revealed that the meningocele was firmly anchored to part of the diaphragm, which created stretching tension in the meningocele continuously with exhalation. Once detached, the meningocele shrank spontaneously and never developed again after cauterization. In this case, continuous or pulsatile pressure in the presence of a vertebral defect was thus considered to be an important factor for formation of the thoracic meningocele.

http://thejns.org/doi/abs/10.3171/2016.2.PEDS15598

KEY WORDS lumbo-costo-vertebral syndrome; thoracic meningocele; pediatric; mechanism; spine

$\mathrm{L}$ UMBO-COSTO-VERTEBRAL syndrome (LCVS) is a rare disorder in children resulting from a single somatic defect at 2-5 weeks of gestational age. It is characterized by hemivertebrae, congenital absence of ribs, meningocele, and hypoplasia of the trunk and abdominal wall presenting as congenital lumbar hernia., ${ }^{2,10,14}$

Here, we report a case of lateral thoracic meningocele in an infant with scoliosis and lumbar hernia, both of which required early surgical intervention. The meningocele was attached to the diaphragm, thus receiving tension through exhalation, which may explain its development.

\section{Case Report}

A 12-month-old girl was referred to our hospital with soft swelling on her left middle back and scoliosis that had been present since birth (Fig. 1 upper). The patient was otherwise healthy with normal delivery and development. She was able to crawl at the time of admission but was not able to walk, and she showed no muscle weakness. Senso- ry examination indicated intact touch and pain sensation, and no abnormal reflexes were found.

MRI of the patient's spine showed a thoracic meningocele protruding to the left lateral side on the T-12 vertebra and syrinx at the same level (Fig. 2A). Ectopia of the spleen was found, which suggested a lack of the posterior abdominal wall at the site (Fig. 2B). The diaphragm was located rostral to the meningocele, which was located outside the thoracic cavity. The meningocele was adjacent to the spleen, and adhesion of the meningocele to the spleen was suspected (Fig. 2C).

CT scans showed multiple anomalies in the thoracic vertebral columns, such as splitting at T-3, T-5, and T-6; fusion of T-8 and T-9; and hemivertebrae at T-10 and T-11. Defects of the left ninth, tenth, and eleventh ribs and fusion of the right first to third and ninth to eleventh ribs were also found (Fig. 1 lower). The meningocele was apparent on the thoracic vertebral column between the eighth and twelfth ribs. Scoliosis was observed with a Cobb angle of $26^{\circ}$. 

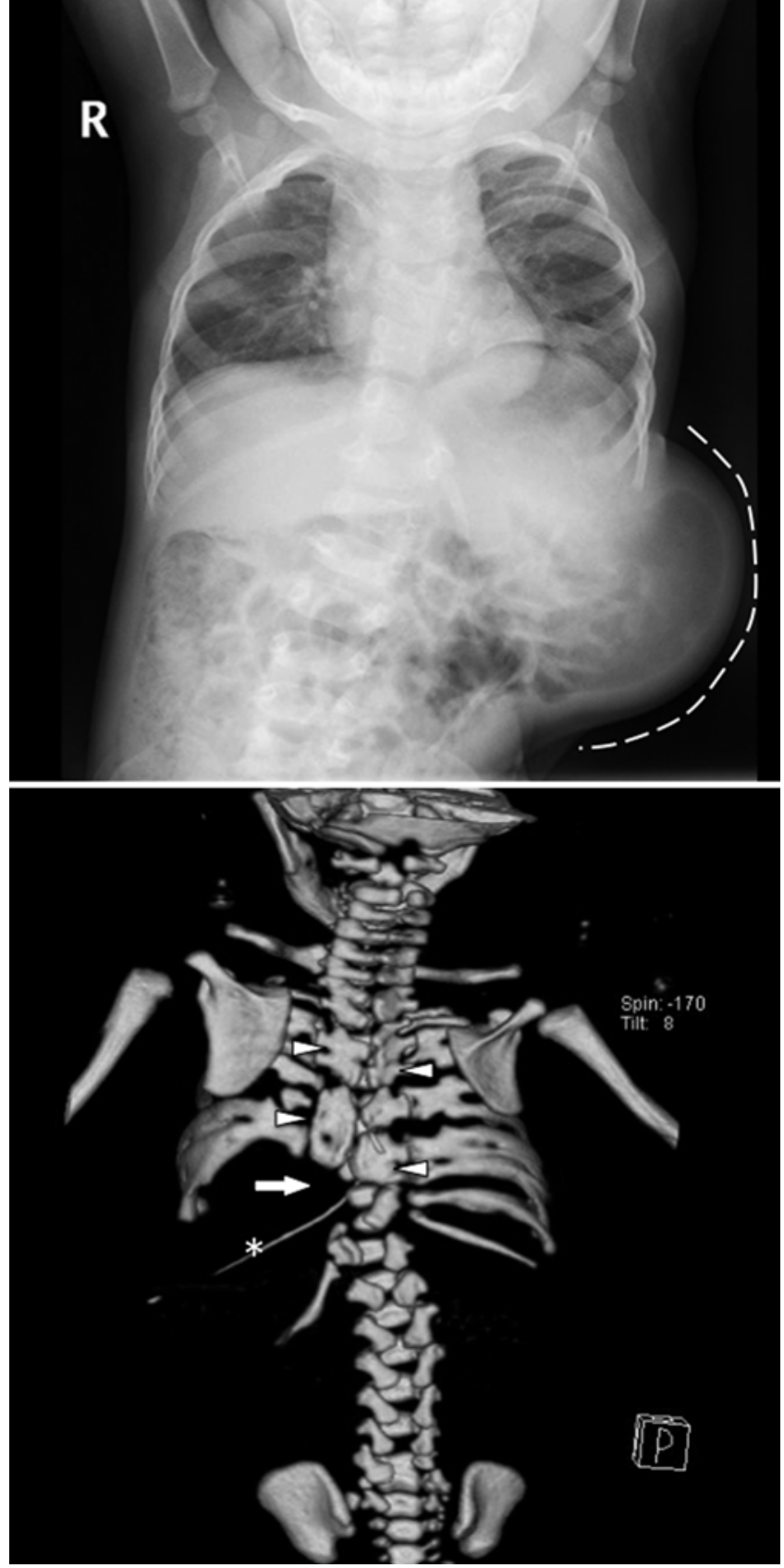

FIG. 1. Upper: Radiograph of the chest and abdomen showing a costal defect and hemivertebrae. A left-sided lumbar hernia is evident (dotted line). Lower: Posterior view of a 3D reconstructed CT scan showing fusion of the spinal column at multiple levels (arrowheads) and hemivertebrae (arrow). Note fusion, defect, and abnormal configuration of the ribs. The asterisk indicates the nasogastric tube.

The general surgery team was planning to repair the lumbar hernia via laparoscopic surgery. However, tight adhesion of the meningocele to the spleen, which could result in significant operative difficulty, was suspected on MRI. Therefore, detachment of the meningocele from the spleen and surgical repair of the meningocele were planned by the neurosurgery team before the procedure to repair the lumbar hernia.
The patient was placed prone, general intubated anesthesia was induced, and a 4-cm midline longitudinal skin incision was made between the posterior spinous processes of T-9 and T-12. The paraspinal muscle was elevated from the spinous process on the left side, but very thin muscle was found at the level of the hemivertebrae. The thin and transparent meningocele wall was easily detected and exposed. The spleen was located just lateral to the meningocele, but no adhesion was observed between the meningocele and the spleen. However, the meningocele was tightly attached to the surrounding tissue on the rostral side by a fibrous band, which was considered to be a part of the left crus of the diaphragm. As a result, the meningocele was stretched with each exhalation (Fig. 3). When the diaphragm was completely detached, the meningocele started to shrink spontaneously and showed less tension. After full exposure, the meningocele was cauterized with low heat to further shrink it until it was finally small enough to fit in the vertebral column. The meningocele was considered to have been mainly caused by the tension generated by the movement of the diaphragm, and therefore no reinforcement or repair of the vertebral defect was applied after the diaphragm was detached. The lumbar hernia surgery was considered to be relatively urgent, and intradural exploration was therefore not performed to avoid surgical complications such as pseudomeningocele that could potentially delay the hernia surgery. The patient subsequently underwent lumbar hernia repair by laparoscopy 2 weeks later, as initially planned.

Preoperative MRI showed mild syrinx at the level of the thoracic meningocele. The meningocele and associated scoliosis were initially considered to be the main reasons for the syrinx, because no obvious tethering structure was found on MRI. However, worsening of the syrinx was observed even after surgery. Thus, a fatty filum terminale and low-lying conus medullaris were considered to be the next candidates for the causative lesion, and incision of the filum terminale, which was considered to be a relatively low-risk procedure, was performed 5 months after initial surgery.

Postoperative MR images obtained 2 years after surgery showed successful repair with no mass effect and no recurrence of the thoracic meningocele (Fig. 2D). In contrast, the syrinx showed interval enlargement even after the surgeries, although no symptoms were apparent. MRI indicated a possible membranous intradural tethering structure at the level of the meningocele, which might have caused the syrinx. Because the syrinx remains asymptomatic, the patient is currently under close observation without surgery. She may eventually undergo treatment at our multidisciplinary spina bifida clinic to check her gait and bowel and bladder function. Her scoliosis showed improvement after surgery, although careful observation and tracking of her growth are still required (Fig. 2B).

\section{Discussion}

LCVS is a rare combination of congenital abnormalities and only a few cases have been reported thus far., ${ }^{1,2,4-}$ $6,8,10,12,14$ Special consideration of each case is required for effective treatment. ${ }^{2,10,14}$ Thoracic meningocele is a cystic sac formed from the spinal meninges and usually arises 

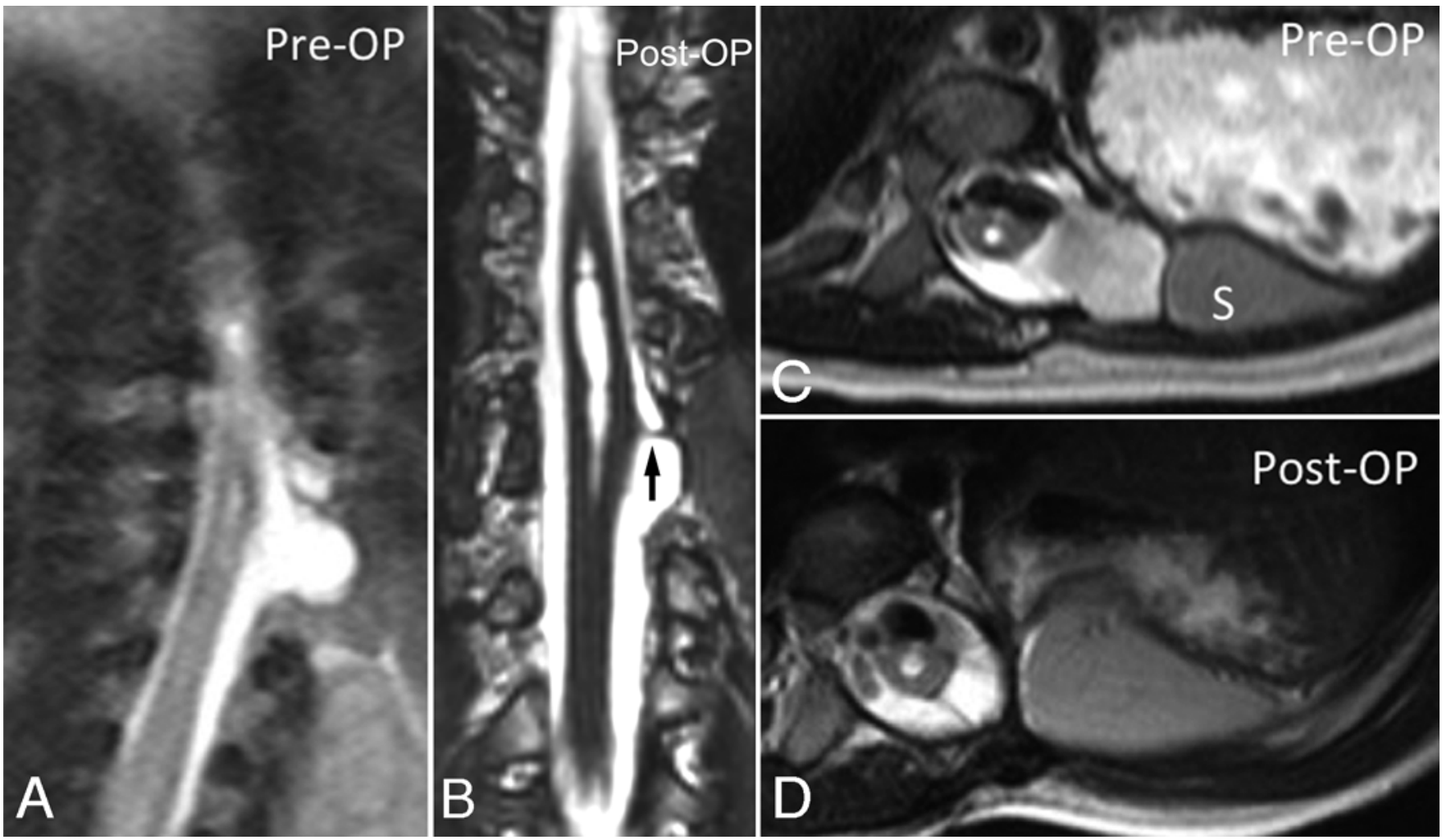

FIG. 2. T2-weighted MR images obtained before ( $A$ and $\mathbf{C}$ ) and after (B and $\mathbf{D})$ surgery. Postoperative MRI indicates development of syrinx and an intradural membranous structure (arrow in B). Tight attachment and adhesion to the spleen (S) of the meningocele was suspected before surgery $(C)$. No postoperative meningocele growth is observed for 2 years after surgery $(B$ and $D)$.

in the presence of an enlarged intervertebral foramen or hemivertebrae. Most reported cases are associated with neurofibromatosis Type 1 ; cases without neurofibromatosis Type 1 are extremely rare. .,11,13 $^{3}$

Thoracic meningoceles are found incidentally and without clinical symptoms in most cases, and the indication for surgery should be carefully considered, especially when there are no symptoms. ${ }^{11}$ The literature even recommends that analgesic treatment should be attempted prior to consideration of surgical repair because of the potentially high risk of the surgical procedure, and only patients with a high degree of discomfort are recommended for surgery. ${ }^{11}$ In the present case, the congenital lumbar hernia already required surgical repair. ${ }^{12}$ To maximize the safety of the endoscopic treatment, surgical exploration was recommended to determine whether the meningocele was adherent to the spleen. The continued growth of the meningocele was also considered to be an indication for surgery.

The muscular attachments of the eleventh and twelfth ribs play an important role in strengthening the region, and abnormality of the eleventh and twelfth rib structures results in weakened attachments of the involved adjacent musculature. This weakness predisposes patients with LCVS to lumbar hernia. ${ }^{1}$ An abdominal hernia was recently safely treated using laparoscopic surgery, and lumbar hernia can theoretically be treated using a similar method. ${ }^{6,8}$ In our case, the association between meningocele and the adjacent organs needed to be carefully determined before and during surgery. Once the meningocele

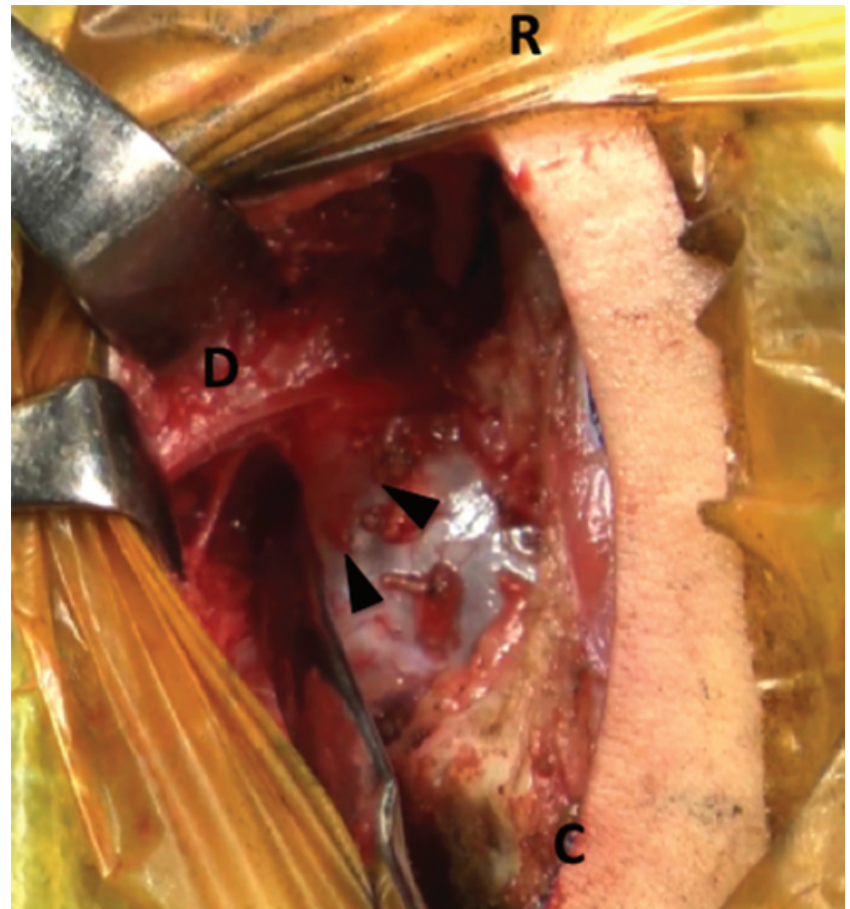

FIG. 3. A fibrous band is tightly anchored (arrowheads) to the meningocele. The capsule of the meningocele was stretched along with the diaphragm during breathing. $C=$ caudal side; $D=$ diaphragm; $R=$ rostral side. Figure is available in color online only. 
was detached and repaired, the lumbar hernia was treated successfully under laparoscopic surgery through the typical procedure.

The present patient exhibited scoliosis before surgery, and she showed improvement after the meningocele surgery without repair of the spinal column deformity. Scoliosis caused by hemivertebrae usually bends toward the side of the vertebral defect. The scoliosis in our patient, however, was bending toward the other side. Therefore, her scoliosis was considered to be formed secondary to the mass effect of the meningocele. The laminae of the vertebrae are fused at multiple levels, which makes prediction of the prognosis difficult; additionally, the laminar fusion may prevent the worsening of hemivertebraederived scoliosis by structurally reinforcing the vertebrae. Therefore, vertebral repair was not performed at the time of the first surgery.

Different etiologies can explain the different developmental mechanisms of meningocele among patients. If the patient has a history of meningeal nerve sheath trauma, subsequent loculation of the escaped CSF can result in progression..$^{13}$ For patients without a history of trauma, the pulsating motion and pressure of the CSF are considered to play an important role in meningocele formation, especially when preexisting dural weakness or bony defects are present. ${ }^{11}$ Another hypothesis specific to the thoracic region is that negative intrathoracic pressure promotes the development of meningocele. ${ }^{7,911}$ Both hypotheses are essentially identical in that continuous pulsatile pressure and vertebral opening are essential for formation of thoracic meningocele. Although pathological evidence to support these hypotheses is lacking, we consider that the theoretical basis is sound.

In the present case, the thoracic meningocele was located outside the thoracic cavity, and the negative pressure theory could therefore not be applied. However, the meningocele was attached to the crus of the diaphragm and thus anchored to it, thereby creating stretching tension continuously with respiratory movements. It is possible that this anatomical relationship was responsible for the development of the meningocele, and our case might therefore support the hypothesis that continuous or pulsatile pressure is responsible for the formation of a thoracic meningocele.

For treatment, the meningocele showed shrinkage after simple detachment, and the stretching movement of the meningocele with breathing disappeared. There has been no regrowth of the meningocele without reconstructions of vertebral anomaly thus far at 2 years after surgery, although continued careful observation is still necessary.

\section{Conclusions}

A rare case of pediatric LCVS with a potentially new underlying mechanism was presented. Continuous pulsatile tension caused by anchoring to the crus of the diaphragm was considered to be potentially responsible for thoracic meningocele formation.

\section{Acknowledgments}

We are grateful to Drs. Devang Thakor and Yoshinori Maki for invaluable comments and discussions.

\section{References}

1. Akçora B, Temiz A, Babayiğit C: A different type of congenital lumbar hernia associated with the lumbocostovertebral syndrome. J Pediatr Surg 43:e21-e23, 2008

2. Bhat RY, Greenough A, Rafferty GF, Patel S, Chandler C: Assessment of diaphragm function in lumbocostovertebral syndrome. Eur J Pediatr 163:694-695, 2004

3. Booth AE: Lateral thoracic meningocele. J Neurol Neurosurg Psychiatry 32:111-115, 1969

4. Hancock BJ, Wiseman NE: Incarcerated congenital lumbar hernia associated with the lumbocostovertebral syndrome. J Pediatr Surg 23:782-783, 1988

5. Harris K, Dorn C, Bloom B: Lumbocostovertebral syndrome with associated VACTERL anomalad: a neonatal case report. J Perinatol 29:826-827, 2009

6. Jones SL, Thomas I, Hamill J: Laparoscopic lumbar hernia repair in a child with lumbocostovertebral syndrome. J Laparoendosc Adv Surg Tech A 20:97-98, 2010

7. Leech RW, Olafson RA, Gilbertson RL, Shook DR: Intrathoracic meningocele and vertebral anomalies in a case of neurofibromatosis. Surg Neurol 9:55-57, 1978

8. Lyngdoh TS, Mahalik S, Naredi B, Samujh R, Khanna S: Lumbocostovertebral syndrome with associated VACTERL anomaly. J Pediatr Surg 45:e15-e17, 2010

9. Maiuri F, Corriero G, Giampaglia F, Simonetti L: Lateral thoracic meningocele. Surg Neurol 26:409-412, 1986

10. Okumus N, Bas AY, Demirel N, Zenciroglu A, Surmeli S, Ceylaner S: Lumbocostovertebral syndrome in an infant of a diabetic mother. Am J Med Genet A 152A:1374-1377, 2010

11. Rainov NG, Heidecke V, Burkert W: Thoracic and lumbar meningocele in neurofibromatosis type 1 . Report of two cases and review of the literature. Neurosurg Rev 18:127-134, 1995

12. Sengar M, Manchanda V, Mohta A, Jain V, Das S: Intercostal variant of lumbar hernia in lumbocostovertebral syndrome: our experience with 6 cases. J Pediatr Surg 46:1974-1977, 2011

13. Turgut M, Alhan C, Cihangiroglu M, Topcuoglu MS: Isolated giant intrathoracic meningocele associated with vertebral corpus deformity. Interact Cardiovasc Thorac Surg 3:381383,2004

14. Vagholkar K, Dastoor K: Congenital lumbar hernia with lumbocostovertebral syndrome: a case report and review of the literature. Case Rep Pediatr 2013:532910, 2013

\section{Disclosures}

The authors report no conflict of interest concerning the materials or methods used in this study or the findings specified in this paper.

\section{Author Contributions}

Conception and design: Wataya, Horikawa. Acquisition of data: all authors. Analysis and interpretation of data: Wataya. Drafting the article: Wataya. Critically revising the article: Wataya. Reviewed submitted version of manuscript: Wataya, Horikawa, Kitagawa. Approved the final version of the manuscript on behalf of all authors: Wataya.

\section{Correspondence}

Takafumi Wataya, Department of Neurosurgery, Shizuoka Children's Hospital, 860 Urushiyama, Aoi-ku, Shizuoka 420-8660, Japan. email: watayatakafumi@gmail.com. 